\title{
Vertrag zur prolongierten Beatmungsentwöhnung der AOK Hessen-Methodik und erste Erfahrungen
}

\section{Contract of the German Health Insurance AOK Hessen Concerning Prolonged Weaning-Methods and First Experiences}

Autoren

Dagmar Giese ${ }^{1}$, Jörg Blau², Walter Knüppel ${ }^{3}$, Aggi Neumann-Schiebener ${ }^{4}$, Andreas Günther ${ }^{5,8}$, Christian Reinhardt ${ }^{6}$, Wolfram Windisch ${ }^{7}$, Stefan Andreas ${ }^{6,8}$

\section{(ㄷ) (i) $(;)$}

Institute

1 AOK Hessen

2 Kliniken des Main-Taunus-Kreises, 65719 Hofheim

3 Krankenhaus Bad Arolsen, 34454 Bad Arolsen

4 Asklepios Stadtklinik Bad Wildungen, 34537 Bad Wildungen

5 Agaplesion pneumologische Klinik, 35753 Greifenstein

6 Lungenfachklinik Immenhausen, 34376 Immenhausen

7 Kliniken der Stadt Köln. Lehrstuhl für Pneumologie, Universität Witten/Herdecke, 58448 Witten

8 Bereich Pneumologie, Universitätsmedizin Göttingen, Deutsches Zentrum Lungenforschung (DZL)

Schlüsselwörter

prolongierte Beatmungsentwöhnung, außerklinische Beatmung, häusliche Intensivpflege, Krankenkasse

Key words

prolonged weaning, homecare ventilation, ambulatory intensive care, health insurance company

Bibliografie

DOI https://doi.org/10.1055/a-1207-7731

Online-Publikation: 9.9.2020

Dtsch Med Wochenschr 2020; 145: e108-e115

(c) Georg Thieme Verlag KG, Stuttgart · New York ISSN 0012-0472

Korrespondenzadresse

Prof. Dr. Stefan Andreas

Lungenfachklinik Immenhausen, Pneumologische Lehrklinik, Universitätsmedizin Göttingen, Immenhausen, Germany stefan.andreas@med.uni-goettingen.de

Dagmar Giese

AOK Hessen, Germany

dagmar.giese@he.aok.de

\section{ZUSAMMENFASSUNG}

Hintergrund Die Zahl der Patienten, die im Verlauf einer intensivmedizinischen Behandlung von einer invasiven Beatmung entwöhnt werden müssen, hat in den vergangenen Jahren stetig zugenommen. Ein Teil hiervon bleibt dauerhaft beatmet und muss im ambulanten Bereich betreut werden. Das Gros dieser Patienten ist allerdings nicht in einem erfahrenen Weaningzentrum behandelt worden. Es ist daher wahrscheinlich, dass noch „Weaningpotenzial“ bei einem Teil der Patienten besteht.

Methoden Die AOK Hessen hat nach umfangreicher Analyse Ansatzpunkte zur Verbesserung dieser Situation entwickelt, die hier erstmalig vorgestellt werden. Als wesentlich wurden die fachliche Expertise der das Potenzial einschätzenden Ärzte (Weanigerfahrung bzw. Weaningzentrum), die Option der temporären Unterbringung der Patienten in den neu zu schaffenden Therapiezentren für außerklinische Beatmung (TAB) und die strukturierte ambulante Nachsorge erkannt.

Ergebnisse Bei den TAB handelt es sich um extrastationäre Einheiten, welche einem Weaningzentrum angeschlossen sind. Hier sollen Patienten in wohnlicher Atmosphäre betreut werden, bei denen es bisher nicht gelang, sie von der Invasivbeatmung zu entwöhnen. Der Fokus liegt dabei auf der täglichen physiotherapeutischen und logopädischen Arbeit. Hierfür wird ein Zeitraum von bis zu 6 Monaten ermöglicht, in dem sich die muskuläre und kognitive Situation verbessern soll. Im Anschluss wird dann je nach Verlauf ein erneuter Weaningversuch im Weaningzentrum unternommen.

Diskussion Das Konzept wird im folgenden Artikel ausführlich beschrieben. Erste Erfahrungen zeigen den relevanten Stellenwert der TAB in der Entwöhnung von der Beatmung.

\section{ABSTRACT}

Introduction In recent years, the number of patients requiring mechanical ventilation has increased steadily. In some cases, weaning is not successful. These patients depend on home mechanical ventilation and intensive outpatient care. Surprisingly, most of these patients were never treated in a weaning center. Thus, weaning might still be possible in at least some of them. Health insurance companies have recognized this problem. 
Methods AOK Hessen, a major health insurance company in the German federal state of Hesse, identified some starting points for improvement after having surveyed their patients in ambulant intensive care. Principal points for future measures are expertise of the treating medical center (weaning experience, weaning center), establishment of new centers for homecare ventilation for long term therapy of these patients and a coordinated follow up.

Results Centers for homecare ventilation are wards with a non-hospital atmosphere affiliated to a weaning center. The main focus here is not weaning itself but daily physical and speech therapy. Patients in home care ventilation centers have time (up to 6 months) to improve their physical and mental strength. Afterwards, depending to their development, they can be transferred to the weaning center again.

Discussion In this paper, we introduce the concept of the home care ventilation centers. Initial data suggests that home care ventilation centers can contribute to successful weaning.

\section{Einleitung}

Dank großer Fortschritte in der Intensivmedizin steigt die Zahl der Patienten, die auch bei schweren Erkrankungsverläufen mittels invasiver Beatmung stabilisiert werden können und anschließend dann von der invasiven Beatmung entwöhnt werden müssen, deutlich an [1-3]. Auch bei der AOK Hessen stieg - gemäß kasseninterner Auswertungen - der Anteil der Versicherten mit mindestens einer stationären Beatmungsstunde von 2012 bis 2018 von 8511 auf 9542 Fälle, d. h. um ca. 12 \% an.

Meist können die Patienten nach kurzzeitiger Beatmungstherapie in der Klinik von der Beatmung entwöhnt werden. Angesichts verbesserter intensivmedizinischer Behandlungsmöglichkeiten bei Organversagen, zunehmender Komorbiditäten und der sich verändernden Altersstruktur der Bevölkerung wächst aber die Zahl der Patienten, die nur schwer vom Beatmungsgerät zu entwöhnen sind und deshalb längerfristig beatmet werden müssen [1, 4]. Mit dem Ziel, in den sich etablierenden Weaningzentren eine möglichst hohe Behandlungsqualität zu erreichen, wurde im Jahr 2007 innerhalb der Deutschen Gesellschaft für Pneumologie und Beatmungsmedizin (DGP) das Netzwerk „WeanNet“ gegründet [2, 3]. Eine Auswertung der Datenbank der Deutschen Weaningzentren $(n=11424)$, die nach den Statuten der DGP zertifiziert werden, konnte kürzlich zeigen, dass ca. $21 \%$ der Patienten mit prolongiertem Weaning bei Nichtentwöhnbarkeit in die invasive außerklinische Beatmung verlegt werden [5]. Ca. $3 / 4$ der bei der AOK Hessen versicherten Patienten werden langfristig intensivbehandlungsbedürftig in der häuslichen Umgebung versorgt. Ein Intensivpflegedienst benötigt für die Versorgung eines einzelnen Patienten 5,3-5,5 Pflegefachkräfte. Die monatlichen Kosten hierfür belaufen sich auf ca. $25000 €$. Die Zahl der außerklinisch beatmeten Intensivpflegepatienten stieg in den letzten Jahren in Deutschland wie auch bei der AOK Hessen stark an [1]. Gleichzeitig wurde deutlich, dass das Weaningpotenzial der Patienten nicht systematisch erfasst wird [1, 4]. Wichtig ist hier die Erkenntnis, dass eine invasive Langzeitbeatmung nach erfolglosem prolongiertem Weaning durchaus mit erheblichen Einschränkungen der gesundheitsbezogenen Lebensqualität verbunden sein kann und dass hier mitunter nicht jeder Patient die benötigte Motivation für ein Weiterleben aufgrund der kritischen Begleitumstände aufbringen kann.

In Diskussionen mit Fachgesellschaften wie der DGP sowie einzelnen Experten hat die AOK Hessen die Daten ausführlich analy- siert. Darauf aufbauend wurde in Diskussionen mit einer Vielzahl von Akteuren versucht, auf diese unbefriedigende Situation eine adäquate Antwort zu finden. Der Prozess hat über 2 Jahre in Anspruch genommen. Im Folgenden soll die Ausgangslage, der erarbeitete Lösungsansatz und erste, nichtsystematische Erfahrungen beschrieben werden.

\section{Methoden}

\section{Analyse der bisherigen Situation}

Im Rahmen einer interdisziplinär angelegten Analyse hat die AOK Hessen in den Jahren 2017 und 2018 eine Datenauswertung und Begutachtungen medizinischer Fallunterlagen durchgeführt. Zusätzlich wurde ein intensiver Austausch mit folgenden Gruppen durchgeführt:

- betroffene Patienten und deren An-/Zugehörige,

- Fachärzte, Therapeuten, Geschäftsführer zertifizierter Weaningzentren,

- in der Thematik erfahrene Fachärzte und Therapeuten im Bundesgebiet,

- niedergelassene Ärzte,

- Vertreter von WeanNet und

- andere Krankenkassen.

Durch diese Analyse wurden folgende Feststellungen getroffen:

- Die Zahl der außerklinisch beatmeten Intensivpflegepatienten stieg in den letzten Jahren bei der AOK Hessen stark an

( $\triangleright$ Abb. 1). So war im Zeitraum von 2012 bis 2018 eine Zunahme der im Bereich der außerklinischen Intensivpflege versorgten Versicherten um $70 \%$ zu verzeichnen, die Ausgaben verdreifachten sich in diesem Zeitraum.

- In Hessen gibt es 6 nach WeanNet zertifizierte Weaningzentren. Beatmete oder tracheotomierte Patienten, bei denen hinsichtlich der Beatmungsentwöhnung kurzfristige Fortschritte (ca. innerhalb der nächsten 4 Wochen) zu erwarten sind, können im Anschluss an eine stationäre Behandlung in ein Weaningzentrum verlegt werden. Die Erfolgsquote dieser Weaningzentren übersteigt $50 \%$.

- Nur ein Bruchteil der bei der AOK Hessen versicherten Patienten (ca. 12\%), die im Jahr 2016 aus ca. 80 Kliniken in die auBerklinische intensivpflichtige Versorgung entlassen wurden, 


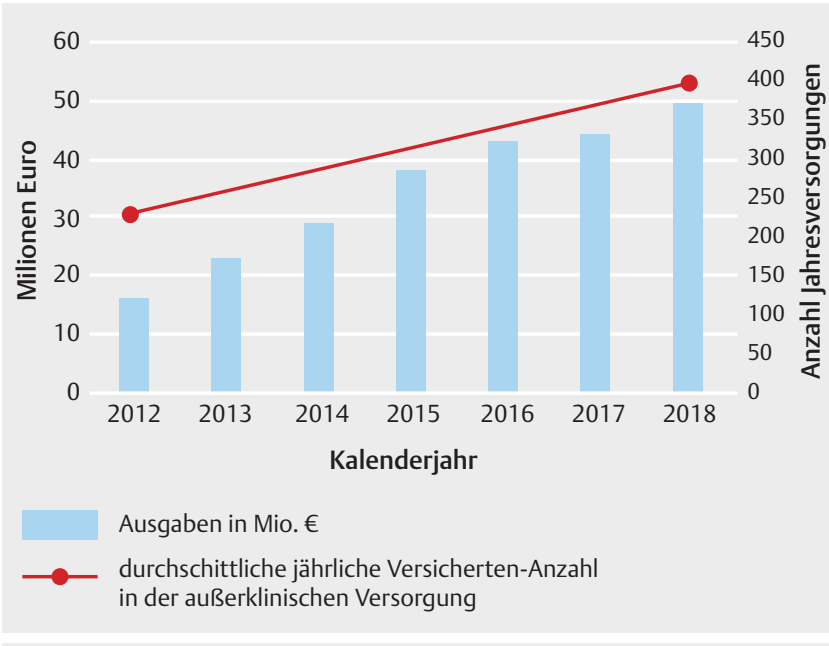

- Abb. 1 Patientenanzahl sowie Ausgaben bei der AOK Hessen im Bereich der intensiven häuslichen Krankenpflege im Zeitraum von 2012 bis 2018.

durchliefen vor der Entlassung in die Häuslichkeit ein zertifiziertes Weaningzentrum.

- Bei den übrigen 88 \% der Patienten erfolgte weder eine Verlegung in ein zertifiziertes Weaningzentrum noch eine Prüfung des Weaningpotenzials durch einen erfahrenen Weaning-Arzt.

- Dass eine Einschätzung eines möglichen Weaningpotenzials durch Fachärzte entlassender Kliniken durchgeführt wurde, war bei Entlassungen oftmals nicht erkennbar. Berichtete Einschätzungen waren teils nicht zutreffend.

- Ein zielgerichtetes Entlass-Management von Akut-Krankenhäusern hin zu Beatmungszentren konnte nicht festgestellt werden. Die gesetzlichen Regelungen des Entlass-Managements wurden nicht regelhaft umgesetzt, da der Kostenträger oft erst nach Einbindung eines Pflegedienstes über die Intensivpflicht informiert wurde.

- Einzelne Pflegedienstmitarbeiter berichteten über ihren Verdacht, dass Verbindungen zwischen einzelnen Klinikmitarbeitern und einzelnen Nachversorgern bestehen, im Rahmen dessen Versorgungsaufträge vermittelt würden.

- Ob und wie Beatmungsentwöhnung in den übrigen Krankenhäusern, bei denen es sich nicht um zertifizierte Weaningzentren handelt, durchgeführt wird, ist wenig transparent.

- Ob der Entlassungs-/Verlegungszeitpunkt beatmeter/tracheotomierter Patienten aus Akutkliniken immer der geeignete ist oder ob eine frühzeitigere Verlegung in ein Weaningzentrum einen besseren Behandlungserfolg hätte herbeiführen können, konnte nicht abschließend geklärt werden.

- Möglichkeiten für ein „Frühwarnsystem“ konnten trotz umfangreicher unterstützender Datenanalysen sowie prozessualer Betrachtungen nicht gesichert erkannt werden. Im aktuellen DRG-System wird die Beatmungspflichtigkeit bzw.

Tracheotomie erst bei der Entlassung aus dem Krankenhaus mitgeteilt, sodass eine gezielte Intervention hinsichtlich Weaning nicht möglich ist. Der Versuch, im Rahmen eines mitarbeitergestützten Klein-Projektes Früherkennungsoptionen mit
Familien zu besprechen, erwies sich nicht als praxisgeeignetes Vorgehen.

- Das DRG-System scheint ökonomische Fehlanreize zu bieten: Die Abrechnung von Beatmungsstunden ist für die Kliniken rentabel, weniger jedoch die erfolgreiche Entwöhnung von der Beatmung.

- In Einzelfällen wurde berichtet, dass das Gelingen des Entwöhnungsprozesses im Weaningzentrum gescheitert sei, da eine Entlassung wegen der Ausschöpfung der maximal abrechenbaren Beatmungsstunden initiiert wurde.

- Die Nachsorge in der Häuslichkeit des Patienten war aufgrund der Trennung des stationären und des ambulanten Sektors schwierig.

- Patienten mit mittelfristigem Weaning-/Dekanülierungspotenzial werden von der Versorgungsmöglichkeit durch ein Weaningzentrum nicht erfasst.

- Eine strukturierte Nachsorge erfolgt in der Häuslichkeit meist nicht.

- Hausärzte sind mit der Betreuung und Behandlung beatmeter oder tracheotomierter Patienten teils unerfahren und unsicher.

- Befragte Experten im Bundesgebiet sahen Weaningpotenziale bei strukturierten erweiterten Versorgungswegen unter unmittelbarer Betreuung und Federführung eines erfahrenen Arztes eines zertifizierten Weaningzentrums. Eine deckungsgleiche Erkenntnis konnte im Rahmen individueller Fallbetrachtungen gewonnen werden.

\section{Ansatzpunkte zur Verbesserung der Versorgungssituation}

Aspekte wie u. a. die Veränderung von Fehlanreizen in der DRGSystematik oder die Etablierung eines „Frühwarnsystems“ wurden zwar als sinnvolle, aber nicht durch die AOK Hessen realisierbare Handlungsfelder erkannt. Folgende Analyseergebnisse hingegen schienen geeignet, positive Veränderungen von Abläufen und Versorgungsmöglichkeiten anzustoßen:

1. Generelles Initiieren der Einschätzung des Weaningpotenzials durch einen erfahrenen Weaning-Facharzt vor der Entlassung in die außerklinische Versorgung.

2. Erweiterung der Versorgungsstruktur um sogenannte TABs (Therapieeinheit außerklinische Beatmung) als Möglichkeit eines mittelfristigen Weaningpotenzials auszuschöpfen.

Die Analyse hatte ergeben, dass die Patienten wochen-, teils monatelang andauernde Klinikaufenthalte hinter sich hatten - und zunächst in ein häusliches Setting überführt werden müssen, in dem sie und ihre An- und Zugehörigen in ihrer Gesamtheit wahrgenommen, betreut und versorgt werden. Eine Haupterkenntnis der Analyse bestand tatsächlich darin, dass das wesentliche Element für den Erfolg der Entwöhnung die „Zeit“ ist. Um den Patienten diese Zeit zu geben, wird eine qualitätsgestützte, erweiterte Versorgungsstruktur benötigt, die wir „Therapiezentrum für außerklinische Beatmung“ (TAB) genannt haben. Die Zielsetzung besteht in der Sicherstellung einer mittelfristigen Beatmungsentwöhnung und Dekanülierung in einem Zeitraum von bis zu 6 Monaten. 
- Tab. 1 Wesentliche Elemente des vertraglichen Rahmens.

\begin{tabular}{|c|c|}
\hline Tätigkeit & Gründe \\
\hline $\begin{array}{l}\text { Vorstellung des Konzeptes } \\
\text { beim Hessischen Ministeri- } \\
\text { um für Soziales und } \\
\text { Integration (HMSI) }\end{array}$ & $\begin{array}{l}\text { - keine Einflussnahme auf den } \\
\text { Landesbettenplan } \\
\text { - Erbitten der Zustimmung des } \\
\text { HMSI }\end{array}$ \\
\hline $\begin{array}{l}\text { europaweite } \\
\text { Ausschreibung }\end{array}$ & $\begin{array}{l}\text { anders als in einem Modellprojekt } \\
\text { ist mit dem IV-Vertrag eine flächen- } \\
\text { deckende Versorgung und der } \\
\text { Abschluss des Vertrages mit } \\
\text { mehreren Kliniken gleichzeitig } \\
\text { möglich }\end{array}$ \\
\hline $\begin{array}{l}\text { Besonderheiten des } \\
\text { Vertrages }\end{array}$ & $\begin{array}{l}\text { - individuelle Vergütung je Klinik } \\
\text { möglich } \\
\text { - Möglichkeit der erneuten } \\
\text { Ausschreibung } \\
\text { - Evaluierung gemeinsam mit den } \\
\text { teilnehmenden Kliniken }\end{array}$ \\
\hline
\end{tabular}

Ein TAB sollte sich durch folgende besondere Kriterien auszeichnen:

- neu zu schaffendes, bisher nicht vorhandenes Versorgungsangebot,

- außerhalb der Räumlichkeiten des stationären Weaningzentrums, in Räumlichkeiten ohne Klinikcharakter,

- in unmittelbarer Nähe zum Weaningzentrum unter ständiger Betreuung eines erfahrenen Weaning-Arztes,

- für Patienten ohne akutstationären Behandlungsbedarf,

- mit dem Schwerpunkt auf Leistungen der Physiotherapie, der Atmungstherapie, der Ergotherapie und der Logopädie (montags bis samstags),

- Personalrotation und/oder Erfahrungsaustausch zwischen Personal des Weaningzentrums und des TAB als Garant für den Einsatz hochqualifizierten und erfahrenen Therapeuten- und Fachpflegepersonals sowie

- die Mitaufnahme von Angehörigen soll möglich sein.

3. Nachsorge in der Häuslichkeit, um den Erfolg zu stabilisieren.

\section{Vertraglicher Rahmen}

Um die gefundenen Ansatzpunkte realisieren zu können, musste ein prozessualer und rechtlicher Rahmen geschaffen werden. Die wesentlichen Elemente sind in $>$ Tab. 1 aufgeführt. Eine externe Evaluation und regelmäßige Treffen zwischen Kliniken und AOK zum Informationsaustausch sollen den Prozess optimieren und stabilisieren.

\section{Vertrag zur integrierten Versorgung}

Mit dem Vertrag der integrierten Versorgung gelingt es, bei Versicherten der AOK Hessen eine verlängerte qualifizierte, inhaltlich neu ausgerichtete und wohnortnahe prolongierte Weaningbehandlung anzubieten. Die wesentlichen Aspekte des Vertrages sind im Behandlungsplan ( $\triangleright$ Abb. 2) dargelegt.

\section{Kernpunkte des Vertrages}

\section{Kernpunkt A des Vertrages}

verstärkte Potenzialeinschätzungen durch erfahrene „WeaningÄrzte“

1. Akutkrankenhäuser sollen - motiviert durch ein Anreizsystem bei allen AOK-Hessen-versicherten Patienten (unter Berücksichtigung datenschutzrechtlicher Regelungen) zum Ende einer stationären Versorgung - per Überleitbogen - relevante Patientendaten an ein am Vertrag teilnehmendes Weaningzentrum übermitteln, um so das Potenzial einer Beatmungsentwöhnung und/oder einer Dekanülierung ermitteln zu können.

2. Bei Bestehen kurz- oder mittelfristigen Potenzials übernimmt das Weaningzentrum die Einschreibung in den IV-Vertrag und nimmt die Patientin/den Patienten auf.

- Als kurzfristig positive Prognose für eine Beatmungsentwöhnung wird in diesem Vertrag jener Entwöhnungsprozess bezeichnet, der derzeit als Regelleistung durch die Inanspruchnahme im Rahmen der Krankenhausbehandlung nach §39 SGB V möglich ist.

- Als mittelfristig positive Prognose für eine Beatmungsentwöhnung wird in diesem Vertrag jener Entwöhnungsprozess bezeichnet, welcher Fortschritte im Entwöhnungsprozess innerhalb der nächsten 6 Monate erwarten lässt.

3. Die kurzfristige leitlinienkonforme Beatmungsentwöhnung und Dekanülierung erfolgt im Weaningzentrum selbst.

4. Wenn

- die Beatmungsentwöhnung/Dekanülierung im Weaningzentrum nicht vollständig durchgeführt werden kann, aber mittelfristiges Potenzial für eine weitergehende Beatmungsentwöhnung/Dekanülierung gesehen wird, oder

- die/der Versicherte vor der Behandlung im Weaningzentrum weitergehender Mobilisation/Stabilisation bedarf, erfolgt die Überleitung in ein „Therapiezentrum für außerklinische Beatmung“ (TAB) für maximal 6 Monate.

Eine Überleitung in das TAB ist für einen Zeitraum von bis zu 4 Wochen auch dann - nach Genehmigung durch die AOK Hessen möglich, wenn die Aufnahme im Therapiezentrum für außerklinische Beatmung als sinnhaft einzuschätzen ist hinsichtlich der Anleitung von An- und Zugehörigen in Vorbereitung auf eine häusliche Versorgung - verbunden mit einer körperlichen, psychischen und seelischen Stabilisierung von Patient und An-/Zugehörigen.

\section{Kernpunkt B des Vertrages}

Schaffen von „Therapiezentren für außerklinische Beatmung“ (TAB) zur Ausweitung des stationären Entwöhnungsprozesses Weaningzentren, die Kapazitäten haben, um in gesonderten Räumlichkeiten den stationären Entwöhnungsprozess auszudehnen, können im Rahmen der integrierten Versorgung die weitere Betreuung durch Weaning-Ärzte sowie Therapien und Pflege in einem „Therapiezentrum für außerklinische Beatmung“ (TAB) zur Verfügung stellen. Die Vergütung dieser Leistungen erfolgt in Form einer Komplexpauschale.

Die mittelfristige Beatmungsentwöhnung wird somit zwar ebenfalls durch ein Krankenhaus, das über ein am Vertrag teilneh- 


\section{Behandlungspfad}

zum Vertrag zur Besonderen Versorgung der prolongierten Beatmungsentwöhnung sowie Dekanülierung der AOK Hessen

Entlassplanung eines beatmeten/tracheotomierten Patienten aus einer Phase A/Phase B

Motiviert durch ein Anreizsystem übermittelt die Klinik einen aussagekräftigen Überleitbogen an ein am IV-Vertrag teilnehmendes Weaning-Zentrum
Vermutung eines

Weaningpotentials bei einem

außerklinisch

versorgten

Intensiv-Patienten

Am Vertrag teilnehmendes Weaningzentrum (WZ) nimmt eine Potentialeinschätzung vor - mit dem Ergebnis:

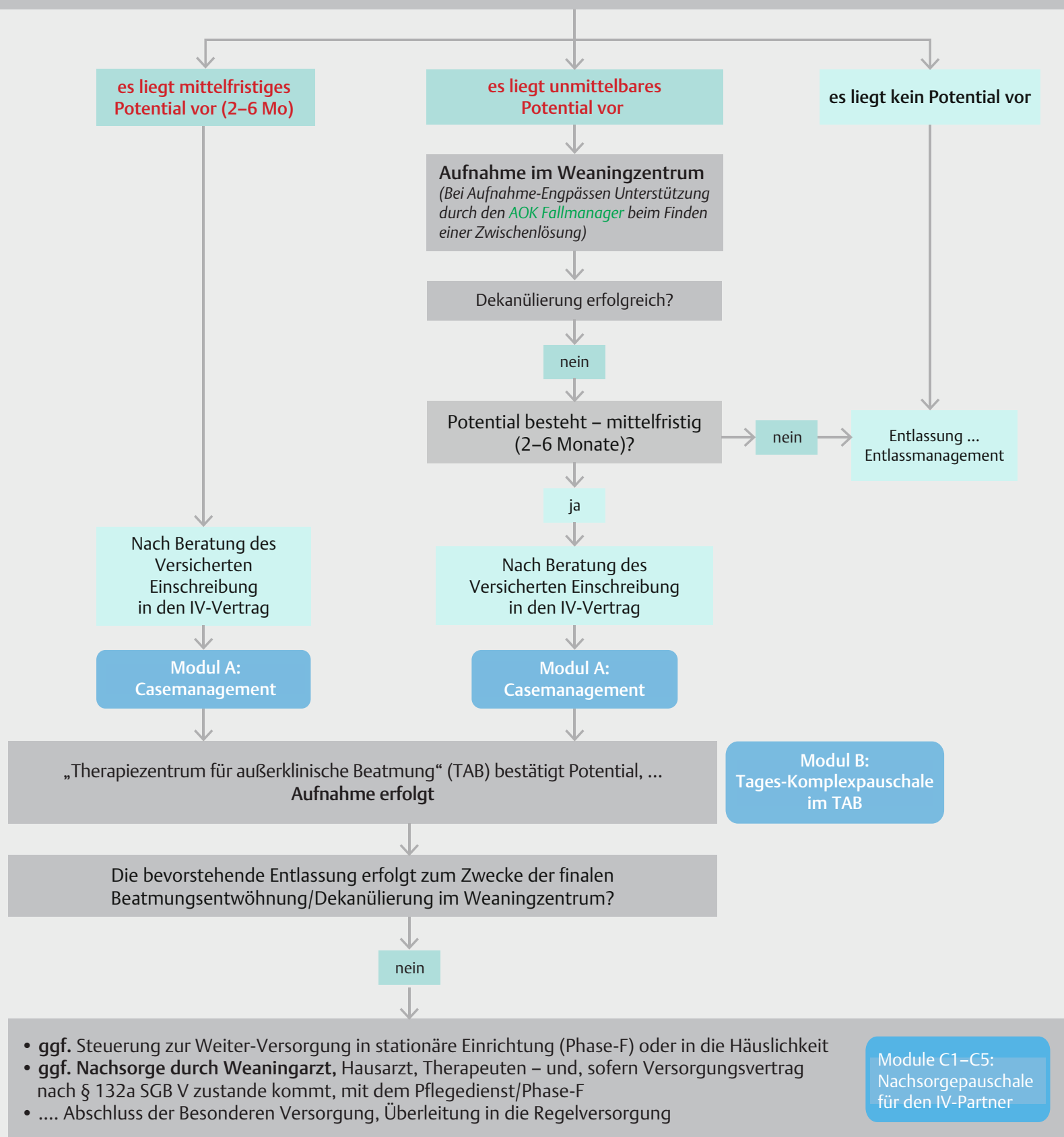

- Abb. 2 Der Behandlungsplan mit den unterschiedlichen Modulen. 
mendes Weaningzentrum verfügt, angeboten - allerdings in einer Behandlungsphase, in der kein akutstationärer Behandlungsbedarf mehr besteht. Die Behandlung selbst erfolgt außerhalb der Räumlichkeiten des stationären Weaningzentrums in einer separaten Station, aber räumlich in unmittelbarer Nähe zum Weaningzentrum, in einem sogenannten $T A B$.

Während der Behandlung im TAB, die bis zu 6 Monate möglich ist, soll der Patient in einer nichtklinischen Atmosphäre stabilisiert sowie durch eine spezielle, individuelle und intensivierte Therapie perspektivisch wieder zum eigenständigen Atmen geführt werden. Die Leitlinien zur prolongierten Beatmungsentwöhnung sind auch hier zu beachten [2, 3]. Insbesondere soll eine enge Kooperation mit dem Weaningzentrum stattfinden, sodass die eigentliche Entwöhnung im Weaningzentrum stattfindet.

Intensive Pflege und individuelle Betreuung des Patienten und auch die der An-/Zugehörigen stehen im Vordergrund. Atmungstherapie, Logopädie, Physio- und Ergotherapien finden von montags bis samstags statt. In der Pflege besteht ein guter Personalschlüssel (maximal 1:3).

Monatlich finden persönliche Fallkonferenzen zwischen AOK und dem TAB statt - zum Austausch sowie zur sehr kurzfristigen Klärung offener Fragen und zur Initiierung und Abstimmung des weiteren Versorgungsgeschehens.

\section{Kernpunkt C des Vertrages:}

\section{Nachsorge}

Sofern das Weaning nicht erfolgreich war, vom Weaning-Arzt aber weiteres Potenzial gesehen wird, findet eine bis zu 1-jährige individuelle Nachsorge statt. Der Weaning-Arzt koordiniert die weitere Steuerung und Betreuung durch das TAB und arbeitet mit dem Hausarzt, dem Pflegedienst sowie Therapeuten zusammen. Im Rahmen eines Modulsystems wird das Schließen von Kooperationsverträgen, die Beratung, das Wiedervorstellen im TAB sowie Hausbesuche durch Klinikpersonal in der Häuslichkeit vergütet. Flexibilität und Individualität sollen durch ein Modulsystem erreicht werden.

\section{Datenschutz, Auswertung}

Bei Aufnahme in das Projekt unterschreiben die Patienten bzw. deren Angehörige eine 4-seitige Teilnahme- und datenschutzrechtliche Einwilligungserklärung, die auch den Befundaustausch zwischen den Behandlern berücksichtigt. Die Einwilligungen können jederzeit ohne Angabe von Gründen widerrufen werden. Die Daten werden nach der Übermittlung anonymisiert, sodass ein Rückschluss auf die Person nicht möglich ist. Die Daten werden spätestens 1 Jahr nach Behandlungsende gelöscht. Die Abrechnungsdaten werden in einer separaten Datenbank gespeichert und nach Ausscheiden aus dem Versorgungsprogramm gelöscht, soweit sie für die Erfüllung der gesetzlichen Anforderungen und Aufbewahrungsfristen nicht mehr benötigt werden.

Die Auswertung der ersten Erfahrungen erfolgt im Rahmen dieser Publikation retrospektiv und deskriptiv. Eine weiterführende, systematische Auswertung wird erst zu einem späteren Zeitpunkt mit aussagekräftiger Datenbasis erfolgen.
- Tab. 2 Darstellung der bisherigen Erfahrungen.

\begin{tabular}{|c|c|}
\hline Kennzahlen & Ergebnisse (Stand Januar 2020) \\
\hline $\begin{array}{l}\text { Anzahl durchgeführter } \\
\text { Potenzialeinschätzungen }\end{array}$ & $\begin{array}{l}67 \text { Versorgungen, davon } \\
\text {. } 75 \% \text { Entlassungen aus Akut-l } \\
\text { Phase-B-Kliniken } \\
\text {. } 25 \% \text { Bestandsversorgungen }\end{array}$ \\
\hline $\begin{array}{l}\text { Ergebnis durchgeführter } \\
\text { Potenzialeinschätzungen }\end{array}$ & $\begin{array}{l}\text { bei } 38 \text { vorgestellten Patienten } \\
\text { wurde ein Potenzial gesehen }\end{array}$ \\
\hline $\begin{array}{l}\text { Anzahl verstorbener } \\
\text { Patienten im TAB }\end{array}$ & 4 Patienten \\
\hline $\begin{array}{l}\text { Anzahl bisher entlassener } \\
\text { Patienten, die im TAB } \\
\text { behandelt worden waren }\end{array}$ & 16 Patienten \\
\hline $\begin{array}{l}\text { Ergebnis der im TAB } \\
\text { behandelten und } \\
\text { entlassenen Patienten }\end{array}$ & $\begin{array}{l}\text { - } 8 \text { Patienten ohne Beatmung und } \\
\text { ohne Trachealkanüle } \\
\text { - } 1 \text { Patient mit NIV (nur nachts, } \\
\text { Versorgung durch die im TAB } \\
\text { angeleitete Familie) } \\
\text { - } 4 \text { Patienten mit Trachealkanüle } \\
\text { ohne Beatmung } \\
\text { - } 3 \text { Patienten mit invasiver } \\
\text { Beatmung }\end{array}$ \\
\hline
\end{tabular}

\section{Ergebnisse/Erfahrungen}

Auch wenn das Konzept erst im Sommer 2019 gestartet wurde, lassen sich bereits jetzt erste Erfahrungen berichten. Über die auch in der Vergangenheit einem Weaningzentrum vorgestellten und dort behandelten Versicherten hinaus sehen die WeaningÄrzte der beteiligten Zentren „on top“ in ca. der Hälfte der vorgestellten Fälle Potenzial für eine Beatmungsentwöhnung/Dekanülierung (siehe $>$ Tab. 2). Ein relevanter Anteil der initial in ein TAB aufgenommenen Patienten konnten im Verlauf im Weaningzentrum von der Beatmung entwöhnt und dekanüliert werden. Ein typischer Patient mit Verlauf wird zur Veranschaulichung in der Fallvignette dargestellt.

Die Rückmeldungen der Versicherten und der An-/Zugehörigen gestalten sich positiv. Es wurde z. B. hervorgehoben, dass die Pflegekräfte und Therapeuten motiviert sind und Zeit für Patienten und Angehörige haben und auf die Ängste sowie die Krankengeschichte der Patienten eingehen. Die individuell gestaltbaren Zimmer und die Aufenthaltsräume geben den Patienten das Gefühl von Häuslichkeit. Dies wird durch einzelne Aussagen unterstrichen: „Der emotionalste Moment war der, in dem ich das erste Mal wieder sprechen konnte." Eine Evaluation mit systematischer Analyse wird durchgeführt werden, wenn Daten über einen längeren Zeitraum vorliegen.

Eine Zusammenfassung der bisherigen Erfahrungen ist in - Tab. 2 dargestellt. 


\section{Fallvignette aus einem TAB}

Patient männlich, 70 Jahre, Aufnahmediagnose: Pneumonie bei vorbestehender COPD (GOLD III).

Die Intubation erfolgte auf der Intensivstation am ersten Tag des KH-Aufenthaltes aufgrund des NIV-Versagens bei ventilatorischer Insuffizienz. Der Patient befand sich insgesamt 111 Tage im Beatmungszentrum. Die Überleitung in das hausinterne Weaningzentrum erfolgte nach 10 Tagen und 2 gescheiterten NIVVersuchen. Die perkutane Tracheotomie erfolgte an Tag 32. Die Aufnahme im TAB erfolgte an Tag 54 mit dem Zielauftrag der Dekanülierung.

Die therapeutischen Maßnahmen wurden entsprechend der Projektbeschreibung, ergänzt durch Hanteltraining und Bettfahrradanwendungen, durchgeführt. Neben der schweren Schluckstörung standen im Vordergrund: Zum einen die allgemeine Kraftminderung (ICU-aquired weakness, ICUAW) - Sitzen im Bett und mit seitlicher Absicherung war möglich -, zum anderen eine massive Angststörung - der Patient hatte kein Zutrauen zu sich und seinem Umfeld. Er sagte bei Aufnahme: „Ich weiß nicht, ob ich das schaffe!" Nur durch die externe Aktivierung seiner Ehefrau kam er überhaupt bis in das TAB. Zwei Aspekte führten im weiteren Verlauf zur Besserung: Erstens führt die intensive Betreuung durch das Pflegepersonal und den Therapeuten zur Wiederkehr des Selbstvertrauens. Zweitens kommt der Patient zur Ruhe. Im Nachgang beschreibt der Patient dies als den wichtigsten Punkt. Das „nervige“ Piepsen und die Unruhe in der Weaningeinheit, die ihn nachts wachgehalten haben, waren weg. In einem Interview, welches der Patient nach seiner Entlassung gegeben hat, sagte er: Er hatte im TAB selbst wieder die Initiative ergriffen und seine Angst verloren.

Der Wechsel auf den Platzhalter (Tag 72) wurde in der Weaningeinheit durchgeführt. Nach 91 Tagen war das Tracheostoma verheilt. Im Rahmen der Fallkonferenz wurde entschieden, den Patienten ohne weitere Rehamaßnahme in die Häuslichkeit zu überführen. Auch dieses Vorgehen motivierte den Patienten. Die Entlassung erfolgte 111 Tage nach Beginn der stationären Behandlung.

Nach der Entlassung aus dem TAB besucht uns der Patient mit seiner Ehefrau wöchentlich und nimmt an dem „Freitags-Talk im TAB“ teil. Hier sitzen unsere Patienten, ihre Angehörigen sowie unser Team und nutzen das psychosoziale Angebot unter Moderation einer ausgebildeten Beraterin. Alle Patienten, die nicht an dem Termin teilnehmen können, besucht er im Zimmer und motiviert sie an dem Ziel zu arbeiten, wieder ein selbstbestimmtes Leben zu erreichen [6].

\section{Diskussion}

\section{Gemeinsame Lösungsansätze}

Ein innovativer Aspekt unseres Projektes ist, dass dieses von einer Krankenkasse nach sorgfältiger Betrachtung der Patientenversorgung unter Einbeziehung von Experten und Betreuungsstrukturen geplant wurde. Hierbei wurde sowohl eine Verbesserung der Patientenversorgung als auch eine Reduktion der Kosten ange- strebt. Ein solches koordiniertes Vorgehen ist im deutschen Gesundheitswesen bei einer Vielzahl von Akteuren nicht alltäglich, sodass Ressourcen nicht immer optimal allokiert werden. Dies wird durch aktuelle Befunde der EU-Kommission bestätigt: In Deutschland liegen die Pro-Kopf-Ausgaben für die Gesundheitsversorgung höher als in allen anderen Mitgliedsstaaten der EU. Die Gesundheitsergebnisse, gemessen an der Lebenserwartung, sind jedoch nur durchschnittlich [7].

Interessanterweise hat unser Projekt Gemeinsamkeiten mit dem geplanten Gesetz zur Stärkung von Rehabilitation und intensivpflegerischer Versorgung in der gesetzlichen Krankenversicherung (Intensivpflege- und Rehabilitationsstärkungsgesetz GKV, IPREG [8]). Z. B. sieht das Gesetzt vor, dass die außerklinische Intensivpflege durch einen für die Versorgung dieser Versicherten besonders qualifizierten Vertragsarzt verordnet werden muss. Auch soll eine rehabilitative Versorgung durch Logopädie, Ergotherapie und Physiotherapie gewährleistet werden.

\section{Qualitätskontrolle durch Einbeziehung der Kasse}

Ein weiterer Aspekt unseres Projektes ist, dass in den regelmäßigen Fallkonferenzen das Behandlungsteam mit Vertretern der Krankenkasse spricht. Bisher sind die Kassen zumeist bei Vergütungsfragen mit den Leistungserbringern in Kontakt. Die regelmäßigen Fallkonferenzen helfen in der Startphase des Projektes, Probleme zu erkennen und gemeinsam zu lösen. Weiter wirken die Fallkonferenzen einer unnötigen Leistungsausdehnung durch z. B. eine Weiterführung der Behandlung bei abzusehender Erfolglosigkeit entgegen. Dies ist relevant, da die zunehmende Kommerzialisierung des Gesundheitssystems in Deutschland Fehlanreize bieten kann [7].

Bisher können die Krankenkassen Qualitätsaspekte bei der Vergütung von Leistungen kaum berücksichtigen. In unserem Projekt war die präzise definierte Strukturqualität das wesentliche Auswahlkriterium. Dieses Beispiel lässt hoffen, dass in Zukunft Qualitätsaspekte bei der Allokation von Ressourcen stärker in den Vordergrund rücken. Versorgungsverträge, die eine verlängerte Entwöhnungsbehandlung ermöglichen, gibt es - lokal begrenzt - auch in Nordrhein-Westfalen.

\section{Erste Erfahrungen}

Die bisher von einzelnen medizinischen Fachgesellschaften aufgezeigten Fehlentwicklungen in der Betreuung von beatmeten Patienten konnten im vorgestellten Projekt nachvollzogen werden. Auch wenn bisher lediglich ein kurzer Zeitraum ausgewertet wurde, konnten wir zeigen, dass ein zusätzlicher Teil der Patienten mit einer außerklinischen, intensivpflichtigen Versorgung erfolgreich von der Beatmung entwöhnt bzw. dekanüliert werden kann, sofern die entsprechenden Versorgungsstrukturen vorliegen.

Unsere Befunde sind von Bedeutung, da sie auf eine große Patientengruppe zutreffen. Im Jahr 2016 sind 23 \% aller stationär aufgenommenen Patienten auf einer Intensivstation behandelt worden [1]. Viele dieser Patienten müssen invasiv beatmet werden und bei $10-20 \%$ dieser Patienten muss eine Beatmung längerfristig fortgesetzt werden. Oft geling eine Entwöhnung von der Beatmung auf der Intensivstation nicht, sodass die Patienten in die außerklinische intensivpflichtige Versorgung entlassen werden. 
Lediglich ein geringer Teil der Patienten wird in ein spezialisiertes Weaningzentrum verlegt. Nach der aktuellen Auswertung des WeanNet-Registers kann die Mehrzahl der Patienten (64,3\%) erfolgreich vom Respirator entwöhnt werden und verlässt das Weaningzentrum ohne invasive Beatmung [2, 5]. Die im Rahmen des hier vorgestellten Projektes erhobenen Daten sind möglicherweise schlechter als die Daten der im Akkreditierungsprozess befindlichen Weaningzentren. Das mag an dem ausgewählten Kollektiv, den über die Jahre immer kränkeren Patienten oder an der noch geringen Fallzahl liegen. Auch kommt es bei der kurzen Beobachtungsdauer zu einer systematischen Unterschätzung des Erfolges.

In unserem Projekt konnten wir sowohl Patienten betreuen, die direkt von einer Intensivstation verlegt wurden, als auch Patienten, die aus der häuslichen Intensivpflege übernommen wurden. Dies zeigt, dass das Projekt gut an die Versorgungsrealität in Deutschland angepasst ist.

\section{Hohe Sterblichkeit und ethische Aspekte}

Soweit bei der kurzen Laufzeit des Projektes zu beurteilen, ist die Sterblichkeit der von uns betreuten Patienten hoch. Dies entspricht Daten aus dem WeanNet sowie internationalen Erfahrungen $[5,9]$. Patienten mit schwerer, chronischer Erkrankung sterben oft an den Folgen ihrer Erkrankung. Diese Möglichkeit wird allerdings selten vorausschauend thematisiert. Patienten, Angehörige sowie Ärzte scheuen verständlicherweise das Thema. Allerdings bessert die „vorausschauende Behandlungsplanung“ die Lebensqualität der betroffenen Patienten und reduziert die Kosten am Ende des Lebens [10]. Gerade Patienten mit häuslicher Beatmung sind oft mit ihrer eingeschränkten Lebenssituation unzufrieden und einige von ihnen wären in Betrachtung dieser Situation lieber gestorben als so zu leben [11]. In einzelnen Fällen ist daher eine Thematisierung der weiteren Behandlung in der TAB oder im Weaningzentrum im Sinne einer vorausschauenden Behandlungsplanung, z. B. im Rahmen einer ethischen Fallberatung,

\section{KERNAUSSAGEN}

1. Die Zahl der Patienten, die im Verlauf einer intensivmedizinischen Behandlung von einer invasiven Beatmung entwöhnt werden müssen, hat in den vergangenen Jahren stetig zugenommen. Ein Teil hiervon bleibt dauerhaft beatmet, was zu einer erheblichen Beeinträchtigung der Lebensqualität führt.

2. Eine frühe Erkennung von geeigneten Patienten mit Weaningpotenzial und der dann täglichen intensiven physiotherapeutischen und logopädischen Arbeit ist in einem geeigneten Vertragsrahmen möglich.

3. Erste Erfahrungen zeigen bei einem relevanten Teil der so behandelten Patienten eine erfolgreiche Entwöhnung von der Beatmung.
Interessenkonflikt

Die Autorinnen/Autoren geben an, dass kein Interessenkonflikt besteht.

\section{Literatur}

[1] Karagiannidis C, Strassmann S, Callegari J et al. Epidemiologische Entwicklung der ausserklinischen Beatmung: Eine rasant zunehmende Herausforderung für die ambulante und stationäre Patientenversorgung. Dtsch Med Wochenschr 2019; 144: e58-e63

[2] Schönhofer B. WeanNet: Das Netzwerk pneumologischer Weaningzentren. Pneumologie 2019; 73: 74-80

[3] Schönhofer B, Geiseler J, Dellweg D et al. Prolongiertes Weaning S2k-Leitlinie herausgegeben von der deutschen Gesellschaft für Pneumologie und Beatmungsmedizin e.V. Pneumologie 2019; 73: 723-814

[4] Köhler D. Explosive Zunahme der häuslichen Krankenpflege bei Beatmeten und Tracheotomierten. Dtsch Med Wochenschr 2019; 144: 282285. doi:10.1055/a-0805-5284

[5] Windisch WDD, Geiseler J, Westhoff $M$ et al. Prolongiertes Weaning von der mechanischen Beatmung: Ergebnisse in spezialisierten Weaningzentren - eine Registerstudie basierend auf der Initiative WeanNet. Dtsch Arztebl Int 2020; in press

[6] RheinmainTV TV. AOK Gesundheitsmagazin im November. In. 25.11.2019 ed; 2019: https://bit.ly/3doqAhf

[7] Korzilius H. Deutsches Gesundheitssystem: Hohe Kosten, durchschnittliche Ergebnisse. Dtsch Arztebl Int 2019; 116: A-2283

[8] Bundesgesundheitsministerium. https://www.bundesgesundheitsminis terium.de/intensivpflege-und-rehabilitationsstaerkungsgesetz.html; In 2019 Stand: 1.7.2020

[9] Jubran A, Grant BJB, Duffner LA et al. Long-Term Outcome after Prolonged Mechanical Ventilation. A Long-Term Acute-Care Hospital Study. Am J Respir Crit Care Med 2019; 199: 1508-1516

[10] Andreas S, Alt-Epping B. Advance care planning in severe COPD: it is time to engage with the future. ERJ Open Res 2018; 4: 00009-2018

[11] Huttmann SE, Magnet FS, Karagiannidis C et al. Quality of life and life satisfaction are severely impaired in patients with long-term invasive ventilation following ICU treatment and unsuccessful weaning. Ann Intensive Care 2018; 8: 38

[12] Smith TA, Disler RT, Jenkins CR et al. Perspectives on advance care planning among patients recently requiring non-invasive ventilation for acute respiratory failure: A qualitative study using thematic analysis. Palliat Med 2017; 31: 566-574

[13] Raskin J, Vermeersch K, Everaerts S et al. Do-not-resuscitate orders as part of advance care planning in patients with COPD. ERJ Open Res 2018; 4: 00116-2017 